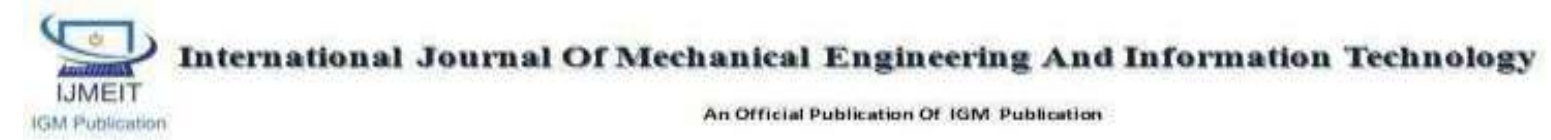

\title{
Vibration Analysis of Railway Bridge Under Moving Loads Using Fluid Viscous Dampers
}

\author{
Authors
}

Ashish Gupta ${ }^{1}$, Prakhar Chansauria ${ }^{2}$, Shailendra Kumar ${ }^{3}$,

${ }^{1,3}$ Department of Mechanical Engineering, IIT Bombay, Powai, Mumbai, India

${ }^{2}$ Department of Mechanical Engineering, MANIT, Bhopal (MP), India

E-mail: ashishiitb99@gmail.com, prakharmahoba@gmail.com, shailendra113.iitb@gmail.com,

\begin{abstract}
Fast trains can induce resonance situations in railway bridges, especially in those where the main structural elements are simply supported beams. Resonance takes place when the time interval between the passages of repeated groups of loads over a certain section of the bridge is a multiple of one of its natural periods. The possibility of mitigating the resonant vibration of simple beams by increasing the overall structural damping with passive energy dissipation devices is evaluated in this paper. More specifically, the proposed the use of linear fluid viscous dampers (FVDs).

We have selected FVDs, as compared to other passive energy dissipation devices, because FVDs dissipate energy at a wide scope of frequency ranges and not only at narrow ranges like TMDs. As a result, they do not present tuning and detuning drawbacks. A control strategy is proposed based on the use of linear fluid viscous dampers that connect the beam carrying the loads (main beam) and an auxiliary beam placed underneath the main one. The main beam equipped with the damping system is subjected to a sinusoidal excitation and analysed with a view to discovering the dampers which minimise the overall dynamic response at resonance. The influence of different bridge parameter is also studied and compared with the help of MATLAB software.
\end{abstract}

Keywords: Railway Bridge; Fluid Viscous dampers; Beam; Resonance; Load, Mass ratio

\section{INTRODUCTION}

Fluid viscous dampers (FVDs) can be used for structural vibration control. Fluid viscous dampers dissipate energy through Fluid volume variation and heat loss by forcing fluid through orifices studied by Zhou Y. al. (2012). Uses of FVD as a means to suppress vertical vibrations in bridges have been conducted. Mitigation of vibrations of short supported railway bridges with the use and implementation of FVD is described by Fry L. (2001). They proposed and evaluated connecting FVD to the bridge slab and an auxiliary structure (a simply supported beam located below the bridge) to control vertical bridge vibration.

\section{CONFIGURATION OF THE DAMPING SYSTEM}

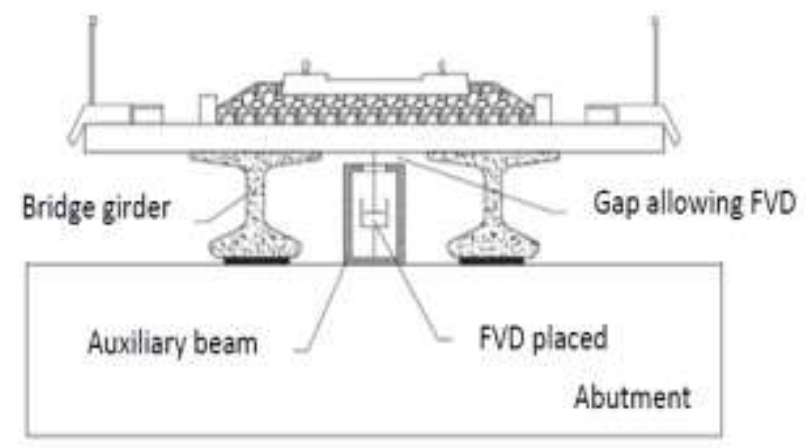

Fig.1 Retrofit configuration for a concrete girder bridge developed by Kwon H.C al. (1998)

The dissipative system to be installed on the beam supporting the passage of the loads consists of two main elements. The first element is an auxiliary, simply supported beam which in typical 
applications is generally located underneath the main beam. The second element is a set of FVDs linking the vertical motion of certain sections of the main beam and the auxiliary one. Our attention here is focused on mitigating the flexural vibrations of the main beam when the train of moving loads induces a resonance situation has been described by Inglis C. E. (1934). For this reason we use a planar model for analyzing the retrofit configuration which is shown in Fig.1

It is assumed that the vehicle-bridge interaction effects are neglected and also the main and auxiliary beams are assumed to rest directly on the abutments without any intermediate elastic bearing.

\section{NON-DIMENSIONAL FORMULATION OF THE BEAM EQUIPPED WITH FLUID VISCOUS DAMPERS}

The system first analyzed under the action of a harmonically varying force. This kind of excitation captures the essential features of the system response at resonance. Assumed that the lengths of both beams are equal and the main beam and auxiliary beam are vertically aligned. These hypotheses are the most relevant ones for real applications If only the first mode of both beams is taken into account, any number of FVDs located at different sections is equivalent to a single equivalent FVD located at mid-span has been described by Oliveto G. al.(1997). Fig.2 shows the configuration of the system with notations.

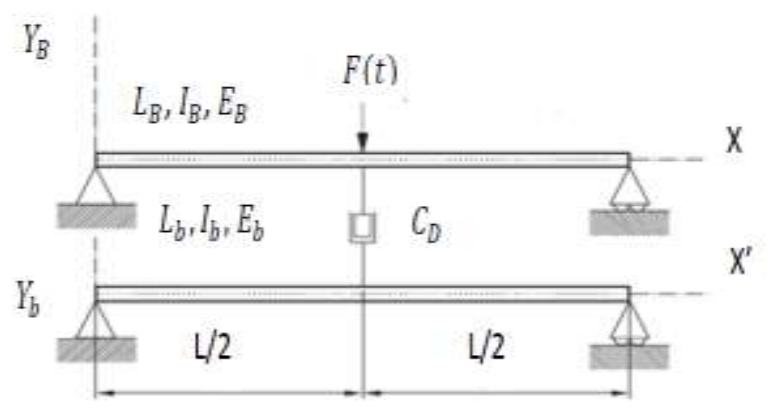

Fig. 2 Represents of retrofitted beam subjected to a sinusoidal load at mid-span
These FVD are to be installed by connecting the beam supporting the passage of the loads (main beam in what follows) to an auxiliary beam, which is most often placed underneath the main one. The system shown is subjected to a point force, which varies harmonically in order to analyze its behavior under resonance conditions.

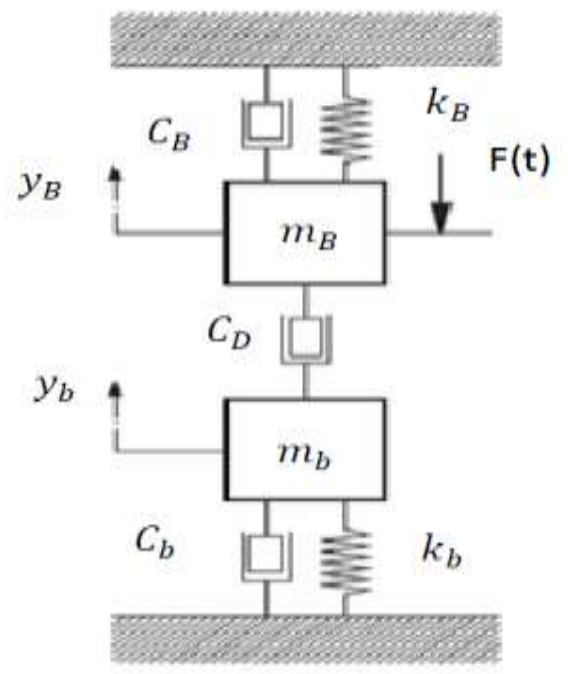

Fig. 3 Represent first flexural modes of vibration of the beams in modal space coordinate

Equations of motions of systems are

$$
M \ddot{y}+C \dot{y}+K=F(t)
$$

Let $\omega_{B}, \omega_{b}$ represent the circular frequencies of the first bending mode of the beams, $\xi_{B}, \xi_{b}$ are the corresponding damping ratios; and $m_{B} L, m_{b} L$ the total masses. $F_{0}, \omega$ are the amplitude and forcing frequency of the excitation, respectively. In order to solve Eq. (1), the following dimensionless ratios are defined.

Frequency ratio

$$
\mathrm{f}=\omega_{b} / \omega_{B}
$$

Excitation frequency ratio

$$
\mathrm{g}={ }^{\omega} / \omega_{B}
$$

Mass ratio

$$
\mu=m_{b} / m_{B}
$$

Supplemental damping ratio

$$
\xi_{D}=\frac{C_{D}}{m_{B} L \omega_{B}}
$$

After some algebraic manipulation, the modal amplifications obtained are

$$
A_{B}=\sqrt{\frac{4 g^{2}\left(\xi_{b} f \mu+\xi_{D}\right)^{2}+\mu^{2}\left(f^{2}-g^{2}\right)^{2}}{E^{2}+4 g^{2} F^{2}}}
$$




$$
A_{b}=\frac{4 g \xi_{D}}{\sqrt{E^{2}+4 g^{2} F^{2}}}
$$

$$
\begin{aligned}
& E=\mu\left(1-g^{2}\right)\left(f^{2}-g^{2}\right)+g^{2}\left(\xi_{D}^{2}+\right. \\
& \begin{array}{l}
\left.\left(\xi_{B}+\xi_{D}\right)\left(\xi_{b} f \mu+\xi_{D}\right)\right) \quad(7 c) \\
\mathrm{F}=
\end{array} \quad\left(1-g^{2}\right)\left(\xi_{b} f \mu+\xi_{D}\right)+\mu\left(\xi_{B}+\xi_{D}\right)\left(f^{2}-\right. \\
& \left.g^{2}\right)
\end{aligned}
$$

Above equations, shows that the amplification of the main beam response $A_{B}$ depends on the following parameters are $\mathrm{g}, \mathrm{f}, \xi_{B}, \xi_{b}, \xi_{D}$. Additionally modal acceleration of the main beam is inversely proportional to its total mass $m_{B} L$. The behavior of the system in terms of these parameters is analyzed in detail in the following section.

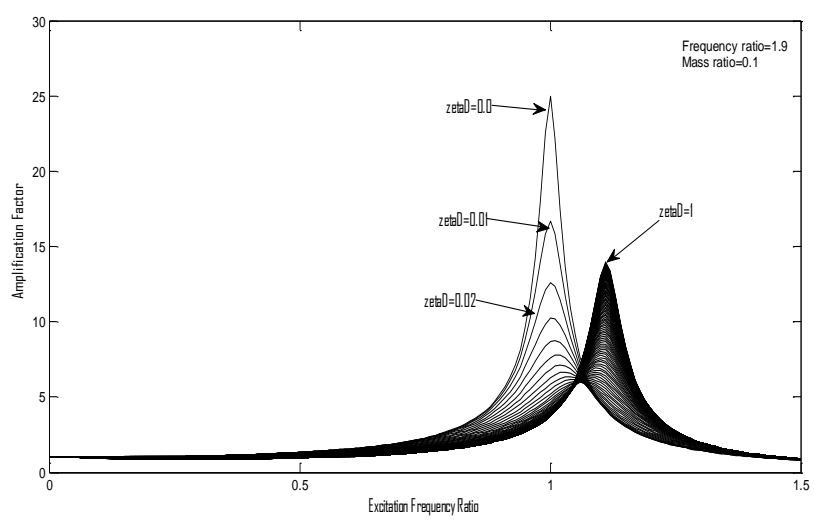

Fig. 4 Amplification of main beam for different values of $\xi_{D}$ for $\mathrm{f}>1$

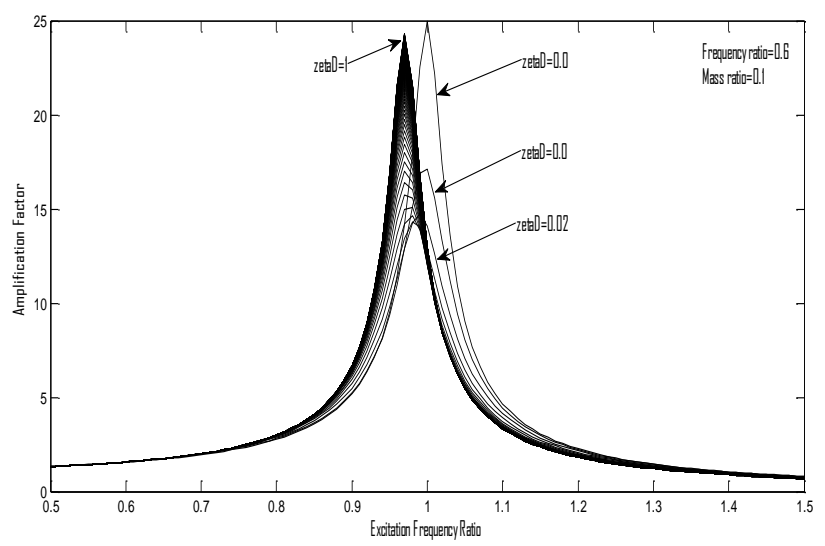

Fig. 5 Amplification of main beam for different values of $\xi_{D}$ for $\mathrm{f}<1$

Fig. $4 \& 5$, shows the dynamic amplification of the main beam for different values of the supplemental damping ratio $\xi_{D}$. In both graphs, $\mu$ is equal to 0.1 . When $C_{D}$ equals zero, there is no interaction and the main beam modal amplitude corresponds to response of a SDOF system. As the damper constant $C_{D}$ increases along with $\xi_{D}$ has been developed by Majka Michal al. (2008). The maximum response decreases, and the value of $g_{R}$ occurs shiftssideways, depends on the value of the frequency ratio. If $\mathrm{f}$ is greater than one, as in Fig. 4, $g_{R}$ increases, and if $\mathrm{f}$ is less than one, as in Fig. 5, $g_{R}$ decreases. The maximum resonant amplification reduces monotonically with $\xi_{D}$ until a minimum value is reached, and increases again if $C_{D}$ keeps increasing. Consequently, for certain values of $\mathrm{f}$ and $\mu$ there is a value of $\xi_{D}$ that leads to the minimum value of the maximum or resonant response. This minimum maximum occurs at a forcing frequency ratio

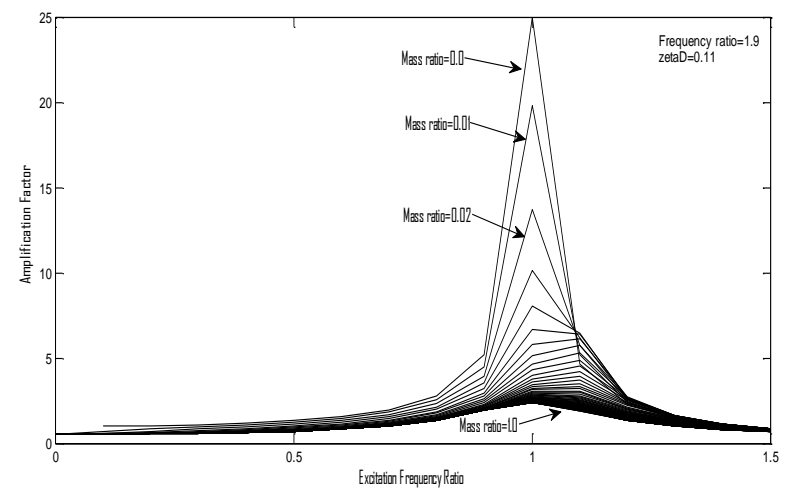

Fig. 6 Amplification of main beam for different values of $\mu$ for $\mathrm{f}=1.9, \xi_{D}=0.11$

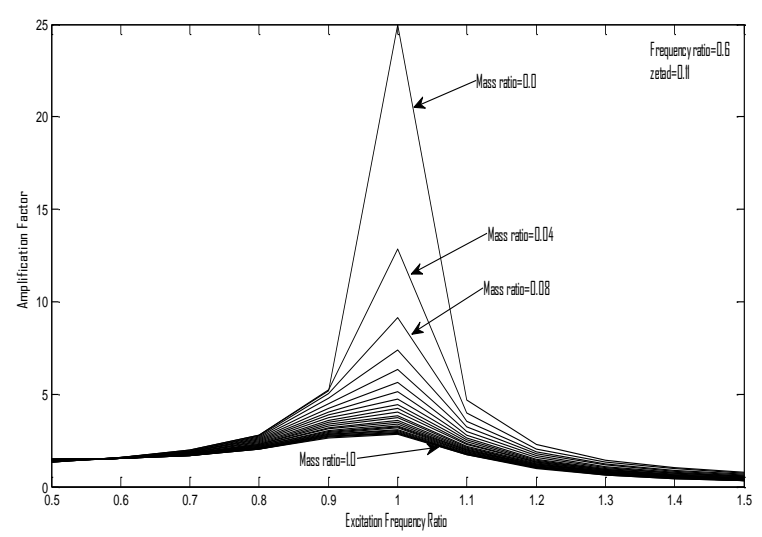

Fig.7 Amplification of main beam for different values of $\mu$ for $\mathrm{f}=0.6, \xi_{D}=0.11$

Fig. $6 \& 7$, gather plots of amplification $A_{B}$ versus $\mathrm{g}$ for a constant value of,$\xi_{D}$ and $\mathrm{f}$ and different values of the mass ratio $\mu\left(\xi_{D}=0.11\right.$ and $\mathrm{f}=1.9 \mathrm{fig}$. 6 , and $\xi_{D}=0.11$ and $\mathrm{f}=0.6$ in fig. 7 . The maximum 
amplification corresponds to a zero value of $\mu$ (i.e. the auxiliary beam is eliminated and the main beam vibrates according to its fundamental mode). As mass ratio increases, the main beam amplification decreases monotonically. The minimum amplification occurs as $\mu$ tends to infinity. In that case, the main beam behaves as if its modal mass were attached through the external damper to a fixed reference, and the dynamic amplification tends to

$$
A_{B}=\frac{1}{\sqrt{\left(1-g^{2}\right)^{2}+4 g^{2}\left(\xi_{B}+\xi_{D}\right)^{2}}}
$$

Moreover, if a certain value of the mass ratio is exceeded, the response becomes almost insensitive to additional increments of this variable. In Fig.6, for instance, no significant change in $A_{B}$ is observed if $\mu$ is given values greater than unity. A similar conclusion is applicable to Fig.7, Besides, as the mass ratio increases, $g_{R}$ shifts to upper values (for $\mathrm{f}>1$ as in fig 4 ) or to lower values (for $\mathrm{f}<1$ as in fig.7).

\section{CONCLUSION}

The damping value of railway bridges has great influence of the amplitude of the response. Higher damping coefficient in the bridge structure gives lower response values. However, the peak values appear at same speed, independent of different damping coefficients.

For a particular auxiliary beam, there exists an optimum value of the FVD constants that minimize the main beam response. The design strategy should be to select the smallest auxiliary beam, which along with its associated optimal damper will provide enough energy dissipation to achieve the desired target performance. With the system proposed in this article the FVDs need not interact with the upper side of the bridge. This allows existing railway bridges to be retrofitted while keeping the line in operation.

\section{Acknowledgments}

The research described in this paper was supported by the Department of Mechanical Engineering, IIT Bombay, India.

\section{REFERENCES}

1. Zhou Y., Lu X., Weng D., Zhang R. (2012), "A practical design method for reinforced concrete structures with viscous dampers", Engineering Structures , 39, 187-198.

2. Fry L. (2001), "A rough assessment of railway bridges for high speed trains", Engineering Structures, 23(5) 548-556.

3. Kwon H.C., Kim M.C., Lee I.W. (1998), "Vibration control of bridges under moving loads", Computers \& Structures, 66(4), 473-480.

4. Inglis C. E. (1934), "A Mathematical Treatise on Vibration in Railway Bridges", University Press Cambridge, England.

5. Oliveto G., Santini A., Tripodi E. (1997), "Complex modal analysis of a flexural vibrating beam with viscous end conditions", Journal of Sound and Vibration, 200(3), 327-345.

6. Majka Michal, Michael Hartnett (2008), "Effects of speed, load and damping on the dynamic response of railway bridges and vehicles", Computers \& Structures, 86(6), 556-572.

\section{Nomenclature}

$\begin{array}{ll}\mathrm{C} & \text { Damping coefficient } \\ \mathrm{E} & \text { Modulus of elasticity } \\ \mathrm{F} & \text { Frequency ratio } \\ \mathrm{L} & \text { Span length } \\ \mathrm{M} & \text { mass per unit length } \\ \mathrm{m}_{\mathrm{a}} & \text { Mass of the bridge } \\ \mathrm{m}_{\mathrm{b}} & \text { Mass of the auxiliary beam } \\ \mathrm{M}_{\mathrm{G}} & \text { Total mass } \\ \mathrm{M} & \text { Mass matrices } \\ \omega & \text { Excitation frequency } \\ \omega_{\mathrm{a}} & \text { Frequency of main mass (i.e bridge) } \\ \omega_{\mathrm{b}} & \text { Frequency of auxiliary mass } \\ \mu & \text { Mass ratio } \\ \xi & \text { Damping ratio } \\ \xi_{D} & \text { Supplemental damping ratio } \\ \xi_{B} & \text { Structural damping of the bridge (i.e main beam) } \\ \xi_{b} & \text { Structural damping of auxiliary beam } \\ A_{B} & \text { Model amplification factor for bridge } \\ A_{b} & \text { Modal amplification factor for auxiliary beam } \\ a_{B} & \text { Modal acceleration of bridge }\end{array}$

